\title{
Repeatable and Transferable Processing for Electron Tomography: An Open Platform for Visualization and Reconstruction of 3D Materials
}

\author{
Robert Hovden ${ }^{1}$, Marcus D. Hanwell ${ }^{2}$, Utkarsh Ayachit ${ }^{2}$, Yi Jiang ${ }^{3}$, Robert Maynard ${ }^{2}$, and \\ David A. Muller ${ }^{1}$ \\ ${ }^{1}$ School of Applied \& Engineering Physics, Cornell University, Ithaca NY, USA \\ ${ }^{2}$ Kitware, Inc., Clifton Park, NY, USA \\ ${ }^{3}$ Department of Physics, Cornell University, Ithaca, NY, USA
}

Three-dimensional (3D) characterization of materials at the nano- and meso-scale has become possible with transmission and scanning transmission electron tomography[1-2]. This process requires advanced software tools where the final 3D visualization is critically dependent on the reconstruction algorithm and the parameters used to render the 3D image. Exacerbating this variability, electron tomography lacks the standards and tools for repeatable, transferable scientific analysis - the field requires open data formats, reconstruction algorithms, 3D visualization, and most importantly a way to share all processing steps from start to finish.

To address this problem, we have developed an open-source platform-tomviz-for the tomographic reconstruction, analysis, and 3D visualization of materials. With a modern graphical interface, tomviz dramatically reduces the barrier of entry to materials tomography in research labs and universities. It is a transparent solution that avoids licensing fees and restrictions on redistribution - allowing researchers at user facilities to processes data off-site.

tomviz can utilize the large quantities of memory and processing resources required to reconstruct, render, manipulate, and analyze voluminous 3D tomograms. The platform provides a robust graphical interface where objects can be rendered as shaded contours or volumetric projections (Figure 1). Multiple datasets, colormaps, and other visualization settings can be used in combination and these objects can be rotated, sliced, animated, and saved as image or video files. 3D data can be further analyzed through histograms, Fourier transforms, and filters - to name a few. The platform is open source, meaning that novel tomographic algorithms or mathematical operations can be readily implemented through its Python (with Numpy) scripting interface or the core $\mathrm{C}^{++}$application (Figure 2).

With tomviz, the full pipeline of data processing steps from reconstruction to visualization and analysis of 3D data can be presented, saved, and restored. This enables fully reproducible results for interlaboratory comparison-critical for fields where researchers share data with colleagues, or raise queries with experts. These pipeline files aid the publication of results, where open scientific methods and peer review are applied to S/TEM materials characterization. tomviz promotes the open Electron Microscopy Dataset specification[3] for large 3D dataset storage.

Reproducible and shareable tomography analysis is necessary for the openness of science and meeting future open-data mandates. tomviz will address a multitude of key issues outlined in the DOE 2013 Data Crosscutting Requirements Review and pursuant to the Executive Order of May 9, 2013, Making Open and Machine Readable the New Default for Government Information.

tomviz is publically available for download at www.tomviz.org

[1] De Rosier, D. and Klug, A. Nature 217, 130-134 (1968)

[2] Midgley, P.A. et al., Chemical Communications, 10, 907-908 (2001)

[3] An Open File Format for Microscopy Data, Based on HDF5, http://emdatasets.lbl.gov/spec/ 

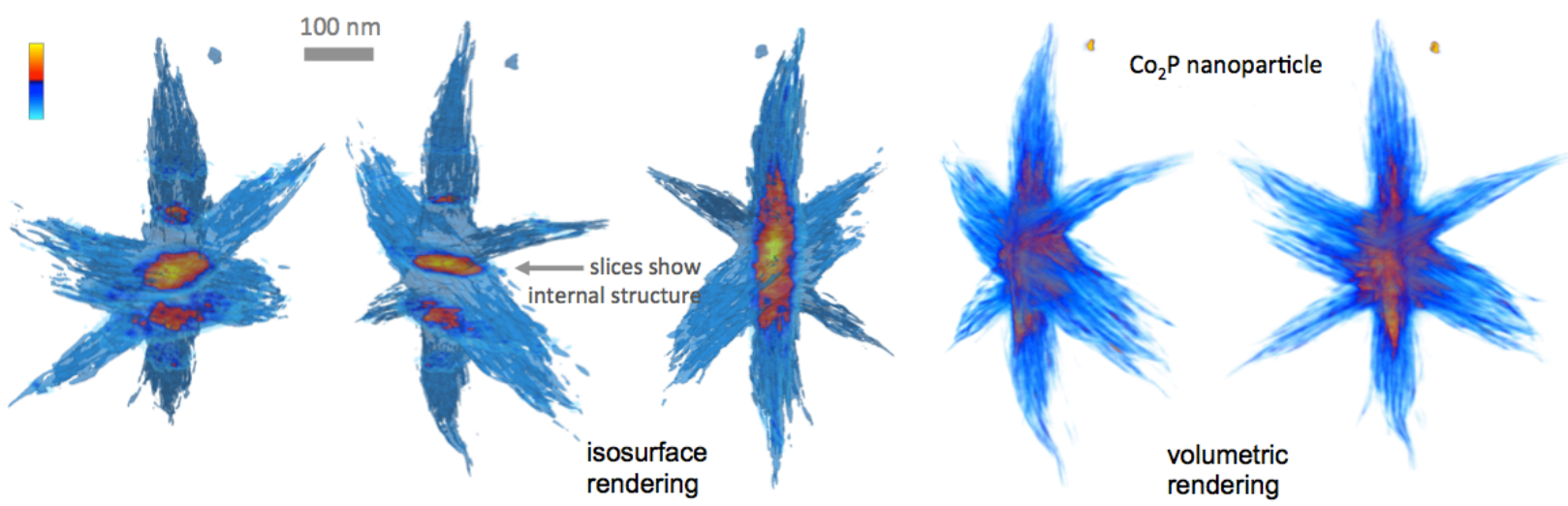

Figure 1 | tomviz 3D visualization of a hyper-branched CoP nanocrystal. An isosurface contours show the particle surface morphology with 2D slices through the particle highlight its high density core region. Volume rendering also shows morphology and changes in particle density.

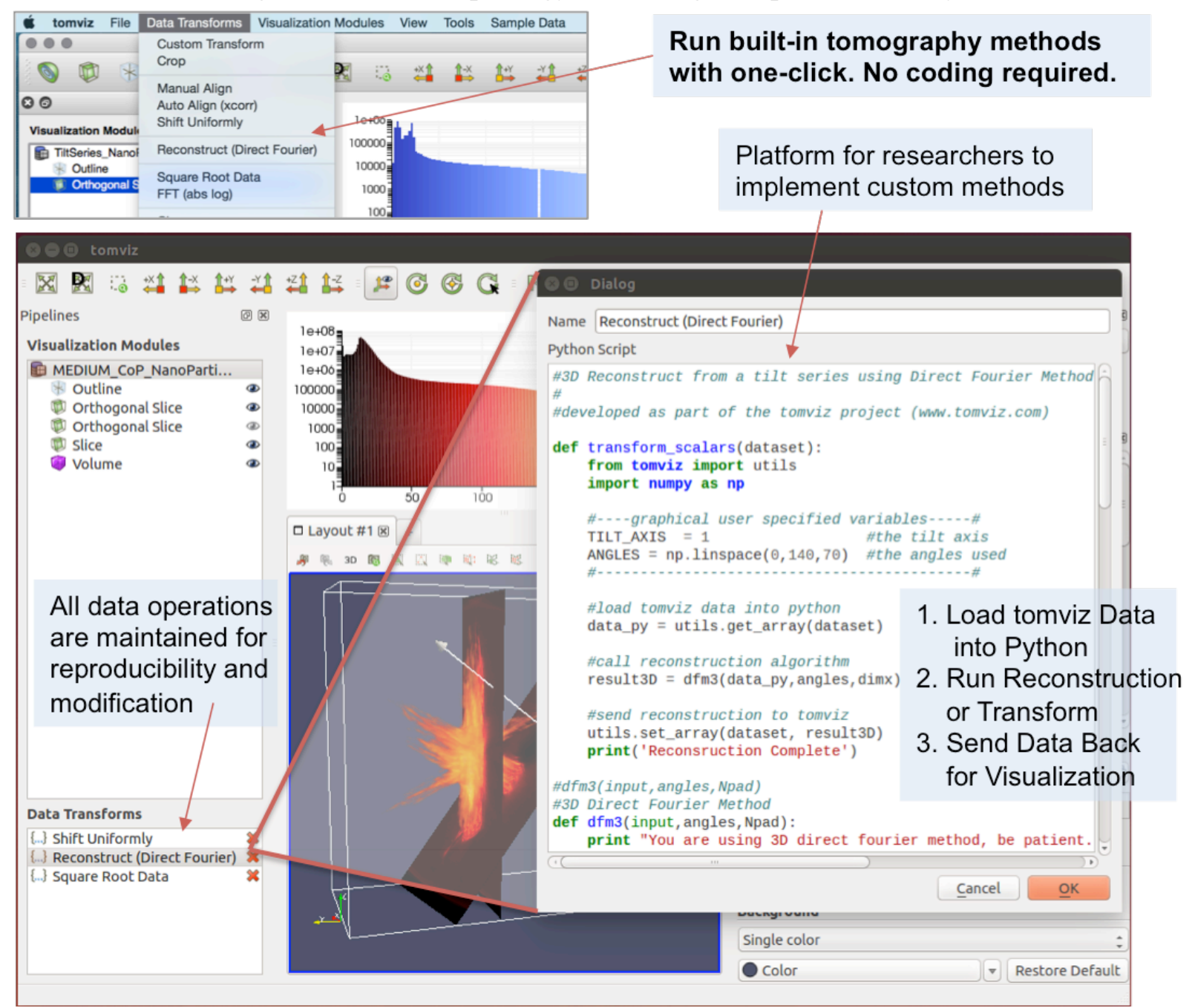

Figure $2 \mid$ tomviz is a graphical platform for tomographic reconstruction and visualization. Reconstruction methods in tomviz are run with a single click; no coding is required. After execution, data is transformed using generated Python code. This code is accessible and can be modified by the user to expand functionality. Through the Python and NumPy interface, custom image processing methods can implemented. The entire analysis and viz pipeline can be saved, restored, and shared. 